\title{
Jahresinhaltsverzeichnis
}

https://doi.org/10.1515/bfp-2017-0051

\section{Die Zukunft des Publizierens}

\section{Ben Kaden und Michael Kleineberg}

Zur Situation des digitalen geisteswissenschaftlichen Publizierens - Erfahrungen aus dem DFG-Projekt „Future Publications in den Humanities“ — 7

Sven Fund

Von der Digitalisierung zur Digitalität: Wissenschaftsverlage vor anderen Herausforderungen -15

Alice Keller

Finanzierungsmodelle für Open-Access-

Zeitschriften -22

\section{Best Practice}

Claudia Büchel

Stadtbücherei Hilden - Bibliothek des Jahres 2016 — 36

\section{Next generation library systems}

\section{Martin Malmsten}

A Step Towards a Distributed Model for Bibliographic Data in Sweden — 40

\section{DARIAH}

Erhard Hinrichs und Thorsten Trippel CLARIN-D: eine Forschungsinfrastruktur für die sprachbasierte Forschung in den Geistes- und Sozialwissenschaften - 45

\section{Weitere Beiträge}

\section{Karl Sand}

Informationsverhalten, sozialer Kontext und Bibliotheken: Annäherungen an Theorien der Small Worlds und Informationswelten $-\mathbf{5 5}$
Frank Frischmuth

Kultur und Wissen digital vermitteln - Stand und Perspektiven der Deutschen Digitalen Bibliothek ein Überblick — 75

Katja Löbel

Bibliotheken auf dem Weg zu lernenden Organisationen Entwicklung eines Selbstbewertungstools — 81

Hans-Joachim Alscher

Die Niederösterreichische Landesbibliothek — 89

Martin Götz

Lernzentren - eine kurze Bestandsaufnahme - $\mathbf{1 0 0}$

Tino Licht und Annelen Ottermann

Aus der Frühzeit der Mainzer Skriptorien: Ein unbekanntes karolingisches Handschriftenfragment (Mainz, Wissenschaftliche Stadtbibliothek, Hs frag 20) - 103

\section{Für die Praxis}

Achim Bonte

Befähigung im Wandel. Personalgewinnung und -entwicklung in deutschen Bibliotheken — 115

\section{Bibliographische Übersichten}

Christoph Albers

Zeitungen in Bibliotheken. Monographien, Aufsätze und Vorträge aus dem Jahr 2015/16 mit Nachträgen aus den beiden Vorjahren - 122

\section{Österreichische Nationalbibliothek}

Christa Bittermann-Wille und Lydia Jammernegg

Das frauen- und geschlechterspezifische Gedächtnis einer Universalbibliothek. Archivierung und Bewahrung, Symbole und Allegorien an der Österreichischen Nationalbibliothek - 140

Michael Hintersonnleitner, Jan Mokre und Christa Müller AKON - Ansichtskarten Online - 149 


\section{Michaela Ortner}

Die Katalogisierung der Bücher der ehemaligen Fideikommissbibliothek des Hauses Habsburg-Lothringen an der Österreichischen Nationalbibliothek — 156

Thomas Zauner

Maria Theresia sagt: Danke! Die Österreichische Nationalbibliothek konnte ihre erste Crowdfunding-Aktion erfolgreich umsetzen — 163

\section{Graz}

Katharina Kocher-Lichem

Die Steiermärkische Landesbibliothek im neuen Joanneumsviertel — 167

\section{Buch- und Literaturmuseen}

Katharina Manojlovic

Labor, Speicher, Bühne: Das Literaturmuseum der

Österreichischen Nationalbibliothek — 175

\section{Bernhard Tuider}

Die Sammlung für Plansprachen und das Esperanto museum der Österreichischen Nationalbibliothek Geschichte, Bestand und Projekte - 185

\section{Heike Gfrereis}

Das ist kein Papier. Die Marbacher

Literaturmuseen -193

\section{Stephanie Jacobs}

Zeichen - Bücher - Netze: Das Deutsche Buch- und Schriftmuseum der Deutschen Nationalbibliothek. Ein Ort der Buch- und Mediengeschichte — 202

\section{Weitere Beiträge}

\section{Elmar Mittler}

Wohin geht die Reise? - Bibliothekspolitik am Anfang des 21. Jahrhunderts - 213

\section{Andreas Degkwitz}

Die Tradition des Textes und die Herausforderung der Daten - 224
Stefan Buddenbohm, Markus Matoni, Stefan Schmunk and Carsten Thiel

Quality Assessment for the Sustainable Provision of Software Components and Digital Research Infrastructures for the Arts and Humanities — 231

John Van Oudenaren

The World Digital Library — 242

\section{Christine Wellems}

Bibliotheks- und Informationseinrichtungen als Partner für eine nachhaltige Entwicklung. Der bibliothekarische Weltverband IFLA und die Agenda 2030 der Vereinten Nationen. Mit einem Interview mit Stuart Hamilton, Director Policy \& Advocacy, IFLA-Zentrale in Den Haag — 250

Yoko Hirose

Use of Private Sector Dynamism in Japanese Public Library: Ebina City Central Library — 257

Karsten Schuldt, Rudolf Mumenthaler und Ekaterina Vardanyan

Schulbibliotheken in der bibliothekarischen Literatur kontra Schulbibliotheken in der Praxis - 262

Sven Bingert, Stefan Buddenbohm, Claudia Engelhardt und Daniel Kurzawe

Herausforderungen und Perspektiven für ein geisteswissenschaftliches Forschungsdatenzentrum — 279

Margo Bargheer and Kizer Walker

Library Publishing and the University Press in the United States and Germany: Lessons from Two Academic Contexts for Sustaining the Scholarly Book — 291

Olga Einasto

The Instrument for Evaluating the Academic Library e-Service Quality — 308

Haike Meinhardt

Leseforschung und ihr Potential für die bibliothekarische Leseförderung — 319

\section{Wilfried Enderle}

Kontinuität der Krise, Krise der Kontinuität? Zur Geschichte wissenschaftlicher Bibliotheken im Nationalsozialismus - $\mathbf{3 3 0}$ 


\section{Schwerpunkt \\ Bestandsmanagement: Last Copies}

\section{Rupert Schaab}

Überlieferung und die beiden Rollen der

Bibliotheken - 353

\section{Pierre Gamache}

Last Copies Initiative: Permanent Conservation of Print

Collections in Canada - $\mathbf{3 6 2}$

John MacColl

Towards a Shared Print Collection in UK Research

Libraries - 367

Ulrich Niederer

Zwei Seiten einer Münze: Die Kooperative Speicher-

bibliothek Schweiz und die Kooperative Print-Archivie-

rung - 375

Bernard F. Reilly

Preserving America's Print Resources - Progress, Challenges and Necessary Measures in North America - 384

\section{Rupert Schaab}

Überlieferungssicherung als Gemeinschaftsaufgabe -

Ein Vorschlag an die Wissenschaftlichen Bibliotheken

Deutschlands - 391

\section{Rezensionen}

Thomas Fuchs

Georg Ruppelt (Hg.): 350 Jahre Gottfried Wilhelm Leibniz

Bibliothek (1665-2015) - 127

\section{Jürgen Geiß-Wunderlich}

Katrin Janz-Wenig, Monika E. Müller, Gregor Patt:

Die mittelalterlichen Handschriften und Fragmente

der Signaturengruppe D in der Universitäts- und

Landesbibliothek Düsseldorf, Teil 1: Textband;

Teil 2: Tafelband - 128
Peter Vodosek

Paul Ladewig: Katechizm biblioteki. Przelożył z języka niemieckiego na język polski Zdzisław Gębolyś; przy wspólpracy Bernharda Kwoki; przelożył z języka niemieckiego na język angielski Zdzislaw Gębolyś — 129

Konrad Umlauf

Frauke Schade (Hrsg.): Praxishandbuch digitale Bibliotheksdienstleistungen: Strategie und Technik der Markenkommunikation -268

Peter Vodosek

Cornelia Briel: Die Bücherlager der Reichstauschstelle. Mit einem Vorwort von Georg Ruppelt — 270

Peter Vodosek

Thomas Eickhoff: Leselust und Stadtkultur in Münster. Hans Thiekötter (1906-1967). Ein Bibliothekar und seine Zeit - 398

Peter Vodosek

Gernot U. Gabel: Bibliotheken in den USA. Beiträge zur Bibliotheksgeschichte. Festgabe zum 75. Geburtstag überreicht von Gisela Gabel-Jahns - $\mathbf{4 0 0}$

\section{Literaturhinweis}

Elmar Mittler

Imprimatur 2017. Ein Jahrbuch für Bücherfreunde. Hg. v. Ute Schneider im Auftrag der Gesellschaft der Bibliophilen in München — 402 
